\title{
ĐÁNH GIÁ CÁC YẾU TỐ ẢNH HƯỞNG ĐẾN HIỆU QUẢ KỸ THUÂTT TIÊM NGOÀI MÀNG CỨNG DƯớI HƯỚNG DẪN CẪT LớP VI TÍNH Ở BỆNH NHÂN THOÁT VỊ ĐĨA ĐỆMCộT SỐNG THẮT LƯNG
}

\section{TÓM TẮT}

Mục đích: Xác định các yếu tố ảnh hưởng đến hiệu quả của kỹ thuật tiêm ngoài màng cứng (NMC) dưới hướng dẫn cắt lớp vi tính (CLVT) ở bệnh nhân (BN) thoát vị đĩa đệm (TVĐĐ) cột sống thắt lưng. Đối tượng và phương pháp: $36 \mathrm{BN}$ đau thắt lưng do TVĐ̋Đ được tiến hành tiêm NMC dưới hướng dẫn CVLT tại khoa X quang can thiệp - Bệnh viện Quân y 103 từ 10/2019 đến 09/2020. Hiệu quả kỹ thuật được đánh giá bằng thang điểm đau VAS (visual analog scales) và thang điểm mất chức năng sinh hoạt $\mathrm{ODI}$ (Oswestry Disability Index). So sánh các yếu tố ảnh hưởng đến hiệu quả kỹ thuật bằng kiểm định Chi bình phương test và $t$ - test. Kết quả: $77,8 \%$ BN hiệu quả tốt, không có $B N$ nào không hiệu quả. Thời gian bị đau và giới tính có liên quan đến hiệu quả kỹ thuật, p $<0,05$. Các yếu tố tuổi, nghề nghiệp, mức độ đau, mức độ mất chức năng sinh hoạt không ảnh hưởng đến hiệu quả kỹ thuật, $p>0,05$. Kết luận: Kỹ thuật tiêm NMC nên được tiến hành sớm ở các BN TVĐĐ từ khi khởi phát triệu chứng đau để nâng cao hiệu quả.

Tư khóa: thoát vị đĩa đệm, tiêm ngoài màng cứng, mức độ đau, mức độ mất chức năng sinh hoạt.

\section{SUMMARY}

THE FACTORS RELATION TO THE EFFECTIVENESS OF CT-GUIDED EPIDURAL STEROID INJECTIONS FOR LOW BACK

PAIN DUE TO LUMBAR DISC HERNIATION

Objects: Evaluating the factors relation to the effectiveness of epidural injection with corticoid under computed tomography guidance for treatment of low back paindue to disc herniation. Subjects and methods: 36 patients with low back pain due to disc herniation were CT - guided epidural injected at the Interventional radiology department, 103 Military Hospitalfrom October 2019 to September 2020. All patients were evaluated by using VAS and ODI scores. Comparisons of the effectiveness with the relative factors were proceeded by $t$ - test and chi square test. Results: The good and moderate effectiveness were $77.8 \%$ and $22.2 \%$, respectively. The long duration of pain and the gender were correlated with the effectiveness. Age, severity of pain and severity of disability were not correlated with the effectiveness. Conclusion: CT-guided epidural spinal injectionsould

*Bệnh viện Quân y 103.

Chịu trách nhiệm: Nguyễn Việt Dũng,

Email: dung.nguyenviet.cdha@gmail.com

Ngày nhận bài: 1/2/2021

Ngày phản biện khoa học: 23/2/2021

Ngày duyệt bài: 25/3/2021
Phùng Anh Tuấn*, Nguyễn Xuân Khái*, Nguyễn Đức Thuận*, Nguyễn Việt Dũng*

be perform as soon as possible for treatment patients with low back pain due to disc herniation.

Keywords: Low back pain, epidural spinal injection, visual analog scale, Oswestry disability index.

\section{I. ĐẶT VẤN ĐỀ}

Trong những năm gần đây, bệnh lý đau thắt lưng do TVĐĐ trở nên phổ biến và gặp cả ở những bệnh nhân (BN) trẻ. Theo nghiên cứu điều tra cộng đồng năm 2015 của Hội Chống đau Hà Nội, trên tổng số 12.136 người ở 48/63 tỉnh, thành phố trong cả nước tỷ lệ người đau thắt lưngchiếm tới 27,75\% [1]. Bệnh thường gặp ở độ tuổi lao động nên việc phát hiện và điêu trị kịp thời có ý nghĩa kinh tế, xã hội to lớn.

Tiêm ngoài màng cứng (NMC) là một biện pháp được áp dụng rộng rãi điều trị giảm đau thắt lưng do TVĐĐ. Mục đích của kỹ thuâat là đưa thuốc chống viêm đến vị trí tổn thương làm giảm quá trình viêm, từ đó giúp giảm đau cho BN. Đây là phương pháp an toàn, mang lại hiệu quả cao. Tuy nhiên, hiệu quả ở những BN khác nhau là không giống nhau. Một số nghiên cứu cho thấy BN lớn tuổi, thời gian bị đau kéo dài, mức độ đau nặng... có liên quan đến việc tăng thời gian hồi phục [3],[5]. Tuy nhiên, mối liên hệ giữa các yếu tố này với hiệu quả kỹ thuật tiêm NMC chưa được đánh giá đầy đủ. Nghiên cứu của chúng tôi được tiến hành với mục đích xác định các yếu tố ảnh hưởng đến hiệu quả kỹ thuật tiêm NMC dưới hướng dẫn cắt lớp vi tính (CLVT) ở BN TVĐĐ cột sống thắt lưng.

\section{II. ĐỐI TƯƠNGG VÀ PHƯƠNG PHÁP NGHIÊN CỨU}

2.1. Đối tượng nghiên cứu. $36 \mathrm{BN}$ khám tại Phòng khám bệnh, Bệnh viên Quân y 103, được chẩn đoán xác định đau thẳt lưng do TVĐĐ và được tiêm NMC dưới hướng dẫn CLVT với tại khoa X Quang can thiệptừ 10/2019 đến 9/2020.

*Tiêu chuẩn lựa chọn:

- BN được chẩn đoán xác định đau thắt lưng do TVĐĐ dựa vào khám lâm sàng kết hợp với hình ảnh cộng hưởng từ ( $\mathrm{CHT}$ ).

- BN có chỉ đinh tiêm NMC: đã điều trị bằng thuốc giảm đau, vật lí trị liệu hoặc kết hợp các phương pháp trên trong thời gian ít nhất 2 tuần nhưng không hiệu quả. 
*Tiêu chuẩn loại trừ: - BN có chống chỉ định chọc NMC: đang có nhiễm trùng vùng tiêm, bệnh lý rối loạn đông máu...

- BN dị ứng với thuốc (thuốc cản quang, thuốc tê tai chỗ, corticosteroid...).

- BN không theo dõi được kết quả điều trị.

\subsection{Phương pháp nghiên cứu}

Tiến cứu, can thiệp không đối chứng, so sánh trước và sau điều trị.

Tiến hành kỹ thuật:

- Thực hiện tại phòng chụp CLVT. Thực hiện trên máy CLVT 2 dãy, nhãn hiệu Somato Spirit, hãng SIEMENS, Đức.

- BN nằm sấp. Chụp CLVT xác định vị trí chọc kim, dự kiến hướng đi kim, độ sâu cần chọc.

- Choc kim, kiểm tra vi trí đâu kim. Tiềm $1 \mathrm{ml}$ dung dich cản quang $10 \%(1 \mathrm{ml}$ Xenetic $+10 \mathrm{ml}$ nước cất), thấy thuốc lan trong khoang NMC.

- Tiến hành block - test bằng $1 \mathrm{ml}$ Lidocain $1 \%$, BN thấy giảm đau rõ rệt

- Tiêm NMC 01 lọ Diprospan 7mg/ml.

Các thang điểm sử dụng trong nghiên cứu

- Thang điểm VAS: Đánh giá mức độ đau theo 11 điểm từ 0 tương ứng không đau đến 10 tương ứng đau không chịu nổi. Từ 1-3: đau nhẹ. 4-7: đau vừa. 8-10: đau năng [7].

- Thang điểm ODI: Đánh giá tổng hợp mức đô mất chức năng sinh hoatdựa trên 10 tiêu chí: Mức độ đau, tự chăm sóc cá nhân, nâng đồ vật, đi bộ, ngồi, đứng, ngủ, hoạt động tình dục, hoạt đông xã hội, đi du lịch. Điểm ODI 0 : hoàn toàn bình thường và 100 mất hoàn toàn chức năng.
Căn cứ vào điểm ODI, tình trạng mất chức năng được chia làm 5 mức độ. Độ 1 (mất chức năng nhẹ): ODI 0-20. Độ 2 (mất chức năng vừa): ODI 21-40. Độ 3 (mất chức năng nặng): ODI 41-60. Đô 4 (mất chức năng rất nặng): ODI 61-80. Đô 5 (mất hoàn toàn chức năng): ODI 81-100 [8].

Đánh giá hiệu quả kỹ thuật: Hiệu quả kỹ thuât được đánh giá bằng so sánh điểm VAS và điểm ODI tai thời điểm sau tiêm 1 tháng (phỏng vấn qua điện thoại) với điểm VAS và ODI trước điều tri.

- Hiêuu quả tốt: Giảm điểm VAS >2 và điểm ODI giảm $>10$.

- Hiệu quả vừa: Điểm VAS và ODI giảm thấp hơn mức hiệu quả tốt nhưng lớn hơn mức không hiệu quả.

- Không hiệu quả: Điểm VAS không giảm và điểm ODI giảm < 4.

Phân tích và xử lý số liệu

- Sử dung phần mềm thống kê SPSS 22.0.

- So sánh tỷ lệ bằng Chi bình phương test, so sánh giá trị trung bình bằng $\mathrm{t}$ - test.

\section{KẾT QUẢ NGHIÊN CứU}

3.1. Đăcc điểm chung của nhóm nghiên cứu

- 36 BN gồm10 nam và 26 nữ, tỷ lệ nữ/nam là $2,6 / 1$.

- Độ tuổi trung bình $50 \pm 11,5$. Đa số BN trong độ tuổi 40 - 60 (72,2\%).

- Tất cả BN đều tiêm 1 lần.

- Hiệu quả tốt $28 \mathrm{BN}(77,8 \%)$, hiệu quả vừa 8 BN (22,2\%). Không có BN nào không hiệu quả.

\subsection{Một số yếu tố ảnh hưởng đến hiệu quả kỹ thuật}

Bảng 1. Một số đặc điểm chung và hiệu quả kỹ thuật $(n=36)$

\begin{tabular}{|c|c|c|c|c|}
\hline \multicolumn{2}{|c|}{ Đặc điếm } & Hiệu quả tốt (28) & Hiệu quả vừa (8) & $\mathbf{p}$ \\
\hline \multirow{3}{*}{ Nhóm tuổi } & $<40$ & $5(17,9)$ & $1(12,5)$ & \multirow{3}{*}{$>0,05^{*}$} \\
\hline & $40-60$ & $21(75)$ & $5(62,5)$ & \\
\hline & $>60$ & $2(7,1)$ & $2(25)$ & \\
\hline \multicolumn{2}{|c|}{ Tuối trung bình } & $48,18 \pm 9,06$ & $55,00 \pm 17,87$ & $>0,05^{* *}$ \\
\hline \multirow{2}{*}{ Giới } & Nam & $11(39,3)$ & $0(0)$ & \multirow{2}{*}{$<0,05^{*}$} \\
\hline & Nữ & $17(60,7)$ & $8(100)$ & \\
\hline \multirow{2}{*}{$\begin{array}{c}\text { Nghề } \\
\text { nghiệp }\end{array}$} & LĐ nặng & $12(42,9)$ & $2(25)$ & \multirow{2}{*}{$>0,05^{*}$} \\
\hline & LĐ nhe & $16(57,1)$ & $6(75)$ & \\
\hline
\end{tabular}

*: Chi binh phương test, **: $t$ - test

Nhận xét: BN nữ hiệu quả điều trị thấp hơn BN nam, khác biệt có ý nghĩa thống kê. Các yếu tố khác không có sự khác biệt.

Bảng 2. Thời gian đau và hiệu quả kỹ thuât $(n=36)$

\begin{tabular}{|c|c|c|c|}
\hline Thời gian đau & Hiệu quả tốt (28) & Hiệu quả vừa (8) & \multirow{2}{*}{ P } \\
\hline$<1$ tháng & $12(42,9)$ & $0(0)$ & \multirow{2}{*}{$<0,05^{*}$} \\
\hline $1-3$ tháng & $5(17,9)$ & $0(0)$ & \\
\hline$>3$ tháng & $11(39,2)$ & $8(100)$ & \\
\hline
\end{tabular}

*:Chi binh phưong test

Nhận xét: BN có thời gian đau càng lâu thì hiệu quả tiêm càng giảm. Sự khác biệt có ý nghĩa thống kê, $p<0,05$. 
Bảng 3. Mức độ đau và hiệu quả kỹ thuật $(n=36)$

\begin{tabular}{|c|c|c|c|c|}
\hline \multicolumn{2}{|c|}{ Đặc điếm } & Hiệu quá tốt (28) & Hiệu quả vừa (8) & p \\
\hline \multirow{2}{*}{ Mức độ đau } & Vừa & $20(71,4)$ & $7(87,5)$ & \multirow{2}{*}{$>0,05^{*}$} \\
\cline { 2 - 5 } & Nặng & $8(28,6)$ & $1(12,5)$ & $>0,05^{* *}$ \\
\hline \multicolumn{2}{|c|}{ Điếm VAS } & $6,18 \pm 0,90$ & $5,88 \pm 0,83$ & $>$ \\
\hline
\end{tabular}

*: Chi binh phương test, **: t-test

Nhận xét: Không có sự khác biệt về hiệu quả tiêm ở các mức độ đau khác nhau. Không có sự khác biệt về điểm VAS giữa hai nhóm hiệu quả tốt và hiệu quả vừa.

Bảng 4. Mức độ hạn chế vận động và kêt quả kỹ thuật $(n=36)$

\begin{tabular}{|c|c|c|c|c|}
\hline \multicolumn{2}{|c|}{ Đăc điếm } & Hiêu quả tốt (28) & Hiêu quả vừa (8) & $\mathbf{p}$ \\
\hline Mức đô mất chức & Năng & $9(32,1)$ & $1(12,5)$ & \multirow{2}{*}{$>0,05^{*}$} \\
\hline năng sinh hoạt & Rất nặng & $19(67,9)$ & $7(87,5)$ & \\
\hline \multicolumn{2}{|c|}{ Điếm ODI } & $64,07 \pm 10,03$ & $66,75 \pm 10,31$ & $>0,05^{* *}$ \\
\hline
\end{tabular}

*: Chi binh phương test, **: $t-$ test

Nhận xét: Không có sự liên quan về hiệu quả tiêm và mức độ mất chức năng sinh hoạt. Không có khác biệt về điểm DOI giữa hai nhóm hiệu quả tốt và hiệu quả vừa.

\section{BÀN LUÂ̂N}

Dựa vào thay đổi điểm VAS và điểm ODI, chúng tôi chia BN sau tiêm thành 3 nhóm: hiệu quả tốt, hiệu quả vừa và không hiệu quả. Theo các tiêu chí đánh giá kết quả của kỹ thuật, có $77,8 \% \mathrm{BN}$ có hiệu quả tốt, $22,2 \% \mathrm{BN}$ có hiệu quả vừa sau tiêm 1 tháng. Không có $B N$ nào không có hiệu quả sau tiêm. Snarr J tổng kết y văn cho thấy có tới $60 \%-84 \%$ các BN tiêm NMC dưới hướng dẫn các phương tiện chẩn đoán hình ảnh có hiệu quả giảm đau rõ. Trong nghiên cứu của Lê Năng Hà Chưởng trên $30 \mathrm{BN}$ bị TVĐĐ được tiêm NMC dưới hướng dẫn CLVT, có $76,6 \%$ $B N$ hiệu quả tốt, $16,7 \%$ hiệu quả vừa và $6,7 \%$ BN không có hiệu quả [2]. Như vâyy, kết quả của chúng tôi cũng tương đương với kết quả các nghiên cứu trong và ngoài nước khác. Tiêm NMC dưới hướng dẫn CLVT là phương pháp có tỷ lệ thành công cao.

Trong nghiên cứu của chúng tôi, không có sự khác biệt có ý nghĩa về các đặc điểm tuổi, nghể nghiệp đến kết quả thực hiện kỹ thuật. Giới tính có liền quan đến hiệu quả kỹ thuật. $B N$ nữ có hiệu quả điều trị thấp hơn BN nam. Lê Năng Hà Chưởng nhận thấy không có mối liên quan giữa tuổi, nghề nghiệp đến hiệu quả của kỹ thuật [2]. Billy GG tiến hành tiêm NMC trên $136 \mathrm{BN}$ dưới hướng dẫn CLVT rút ra kết luận, mức độ giảm đau cũng như thời gian duy trì hiệu quả giảm đau của kỹ thuât không phụ thuộc vào tuổi, giới cũng như nghề nghiệp [3]. Ngược lại, nghiên cứu của Cosgrove JL lại cho kết quả tuối và giới liên quan đến hiệu quả điều trị. BN trẻ tuổi và BN nữ có hiệu quả điều trị cao hơn [5]. Chen $B$ nhận thấy trong số $76 \mathrm{BN}$ bị đau thắt lưng và được theo dõi 122 ngày sau khi tiêm NMC, có $47 \% \mathrm{BN}$ có cải thiện, $28 \%$ không thay đổi và $16 \%$ BN nặng hơn. Hiệu quả kém nhất gặp ở những BN được nhận Bảo hiểm khuyết tật an sinh xã hội hoặc được nhận đền bù lao động nặng nhọc.Tác giả kết luận hiệu quả điều trị có vẻ kém hơn ở những BN làm việc nặng nhọc trong thời gian dài [4]. Ảnh hưởng của những yếu tố này đến kết quả tiêm NMC, do vậy cần có sự nghiên cứu thêm.

Hiệu quả tiêm NMC liên quan mật thiết đênn thời gian bị bệnh của BN. Trong nghiên cứu của chúng tôi, BN có thời gian đau càng lâu, hiệu quả tiêm càng giảm. Khác biệt có ý nghĩa thống kê, $p<0,05$. Điều này có thể được giải thích bởiở những $B N$ đau lưng mạn tính, quá trình viêm dính các tổ chức xung quanhđĩa đệm nhiều hơn và nặng nề hơn, vì vậy tiên lượng về đáp ứng điều trị sẽ thấp hơn các $B N$ đau cấp tính.Theo Billy GG, thời gian bị bệnh càng kéo dài thì hiệu quả điêu trị giảm đau càng thấp. Khi thời gian đau không được điều trị tăng thêm 1 tuần, hiệu quả điều trị giảm thêm $0,07 \%$ [3]. Theo Chen $B$, những $\mathrm{BN}$ mới có triệu chứng đau sẽ có hiệu quả điêu trị tốt hơn những $B N$ đau mãn tính. BN có triệu chứng đau dưới 3 tháng có tỷ lệ đáp ứng với tiêm NMC khoảng $90 \%$. Tỷ lệ này giảm xuống còn $70 \%$ và $50 \%$ ở những $B N$ có triệu chứng đau từ $3-6$ tháng và đau hơn 1 năm [4]. Snarr J cho biết có tới hơn $75 \%$ BN có triệu chứng đau $<3$ tháng điều trị có hiệu quả tốt. Với những $\mathrm{BN}$ đau $>3$ tháng, kết quả điều trị rất thay đổi và giảm rõ. $B N$ mới bị bệnh cũng có hiệu quả giảm đau bền vững hơn những $B N$ đau mãn tính. Kết quả nghiên cứu của chúng tôi cũng như các nghiên cứu trên cho thấy việc tiêm NMC sớm sau khi chẩn đoán xác định BN đau lưng do TVĐĐ là rất cần thiết để đạt được hiệu quả điều trị tối ưu.

Điểm VAS trước tiêm của hai nhóm hiệu quả tốt và hiệu quả vừa lần lượt là $6,18 \pm 0,90$ và 
$5,88 \pm 0,83$ điểm, khác biệt không có ý nghĩa thống kê. Cũng không có sự khác biệt về mức độ đau giữa hai nhóm có hiệu quả tốt và hiệu quả vừa trong nghiên cứu này. Trong số $8 \mathrm{BN}$ có hiệu quả vừa, đa số có điểm VAS ở mức $4-6$ điểm, chỉ ở mức đau vừa. Bên cạnh đó, cũng không thấy có sự liên quan giữa mức độ mất chức năng sinh hoạt và hiệu quả của kỹ thuật. Khác biệt về điểm ODI ở hai nhóm hiệu quả tốt và hiệu quả vừa không có ý nghĩa thống kê, $p$ > 0,05 . Trong nghiên cứu của Lê Năng Hà Chưởng, nhóm $B N$ có hiêu quả điều trị tốt, vừa và không hiệu quả có điểm VAS trung bình lần lượt là 7,7; 7,4 và 8 . Khác biệt không có ý nghĩa thống kê [2]. Nghiên cứu của Park TK trên 150 BN cho thấy hiệu quả tốt của kỹ thuât ở các nhóm điểm ODI $0-30,31-60$ và >60 lần lướt là $61,1 \%$, $73,3 \%$ và $41,6 \%$. Sự khác biệt không có ý nghĩa thống kê [6]. Đánh giá các yếu tố ảnh hưởng đến hiệu quả của tiêm NMC điều tri đau do thoái hóa cột sống thắt lưng, nghiên cứu của Sivaganesan A trên 239 BN nhận thấy các yếu tố hẹp ống sống, thoát vị đĩa đệm và điểm ODI trước điêu trị cao giúp tăng có ý nghĩa hiệu quả điều tri sau 3 tháng. Thời gian đau kéo dài, tiền sử phẩu thuật cột sống là những yếu tố gây giảm hiệu quả điều trị. Kết quả khác biệt giữa các tác giảcho thấy vấn đề này cần có những nghiên cứu thêm.

\section{KẾT LUÂ̂N}

- Tuổi, nghề nghiệp, mức độ đau và mức độ mất chức năng sinh hoạt không ảnh hưởng đên hiệu quả điều trị của kỹ thuật.
- Bệnh nhân nữ có hiệu quả điều trị kém hơn bệnh nhân nam.

- Những bệnh nhân có thời gian xuất hiện triệu chứng đau lâu hơn thường ít đáp ứng với kỹ thuật tiêm ngoài màng cứng.

\section{TÀI LIỆ THAM KHẢO}

1. Nguyen Van Chuong, Dinh Cong Pho, Nguyen Thi Thanh Thuy et al. (2019). Pain incidence, assessment, and management in Vietnam: a crosssectional study of 12,136 respondents. Journal of Pain Research, 12, 769-777.

2. Lê Năng Hà Chưởng (2019). Nghiên cứu hiệu quả giảm đau của kĩ thuật tiêm thẩm phân ngoài màng cứng vùng thắt lưng dưới hướng dẫn cắt lớp vi tính. Luận văn Cao học, Đại học Y Hà Nội.

3. Billy GG, Lin J, Gao M,et àl. (2017). Predictive factors of the effectiveness of caudal epidural steroid injections in managing patients with chronic low back pain and radiculopathy. Clin Spine Surg, 30(6), 833-838.

4. Chen B, Koger T, Mehnert MJ, et al. (2020). Epidural steroid injections.Medscape, https://emedicine.medscape.com/article/325733overview\#a5.

5. Cosgrove $\mathrm{JL}$, Bertolet $\mathrm{M}$, Chase $\mathrm{SL}$, et al. (2011). Epidural steroid injections in the treatment of lumbar spinal stenosis efficacy and predictability of successful response. Am. J. Phys. Med. Rehabil, 90(12), 1050-1055.

6. Park TK, Hong JH, Kim JM, et al. (2008). Factors associated with the outcome of transforaminalepidural steroid injections. Korean J Anesthesiol, 55(3), 298-304.

7. Price DD, McGrath PA, Rafii A, et al. (1983). The validation of visual analogue scales as ratio scale measures for chronic and experimental pain. Pain, 17:45-56.

8. Roland M and Fairbank J (2000). The RolandMorris Disability Questionnaire and the Oswestry Disability Questionnaire. Spine, 25(24), 3115-3124.

\section{ĐÁNH GIÁ KẾT QUẢ ĐÎ̀U TRI GÃY XƯƠNG HÀM DƯớI TẠI BỆNH VIÊ̂N ĐA KHOA SÀI GÒN}

\section{TÓM TẮT}

Mục tiêu: Đánh giá kết quả bước đầu điều trị gãy xương hàm dưới tại Bệnh viện Đa khoa Sài Gòn. Phương pháp: nghiên cứu tiến cứu mô tả hàng loạt ca trên tất cả bệnh nhân có gãy xương hàm dưới được

${ }^{1}$ Dại họ Y Dượ TP.HCM

²Bênh viện Đa khoa Sài Gòn

Chịu trách nhiệm chính: Huỳnh Kim Khang

Email: kimkhanghuynh@yahoo.com

Ngày nhận bài: 2/2/2021

Ngày phản biện khoa hoc: 29/2/2021

Ngày duyệt bài: 21/3/2021

\section{Huỳnh Kim Khang ${ }^{1}$, Nguyễn Hoàng Linh ${ }^{2}$}

điều trị tại khoa Ngoại Tổng hợp bệnh viện đa khoa Sài Gòn từ 1/ 6/ 2015 đến tháng 31/5/2020. Kết quả: Tổng cộng có 76 trường hợp gãy xương hàm dưới được chẩn đoán và điêu trị. $55,3 \%$ có 1 đường gãy. Chẩn thương phối hợp thì gãy tầng mặt giữa chiếm 44,2\%. Chúng tôi thực hiện 54 trường hợp chỉnh hình xương hàm gò má có sử dụng nẹp vít nhỏ và có kết quả tốt. Khớp cắn đúng sau điều tri với tỷ lệ 92,1\% Kết luận: Gãy xương hàm dưới chiếm tỷ lệ cao trong tai nạn giao thông và ẩu đả. Kết quả theo dõi sau phẫu thuật bệnh nhân phục hồi về giải phẫu, chúc năng và thẩm mỹ tốt. Cần nghiên cứu với mẫu lớn và thời gian theo dõi dài để có đánh giá toàn diện. nẹp vít. 